\title{
Coping Mechanisms and Parental Relationship in the Families of a Child with Autism Spectrum Disorder
}

\author{
Smriti Gour ${ }^{1}$, Neelam Pandey ${ }^{2}$
}

\section{ABSTRACT}

Autism Spectrum Disorder (ASD) is a pervasive developmental disorder, which, with increased awareness, is now diagnosed early and with greater accuracy in India. This study aims to assess the coping mechanisms and parental relationship in families with a child having ASD. In depth interviews were conducted for 10 families with 4 members each $(n=40)$. The interviews contained questions for investigating the coping mechanisms, relationship between the parents and the sibling of the child with ASD, separately interviewing the mother, father and the typically developing sibling. The key findings of the interview suggested that the families depended on household help and gathering information on the internet, which, instead of serving as the coping mechanism it was intended to be, led to increased stress for the parents. Other methods included advice and help from professionals and spiritual methods like reading scriptures, family support and the support of the society. The acceptance of the diagnosis and general optimism, along with having specific responses for aberrant behavior defined by the professional were found to be the most effective coping mechanisms for the families. The relationship between parents was stressed in almost all cases, but the families which accepted the diagnosis early and sought the help of professionals, consequently, had a much better and complementary relationship. The families also relied on the typically developing sibling for the care of the autistic child. The relationship between the sibling and the child with ASD was close and the elder sibling, if female, also took on a maternal role in some cases in the caretaking of the child. In the case of male elder sibling, the relationship was close but there was lesser delegation of responsibility for the autistic child by parents.

Keywords: Coping mechanism, Parental relation, Autism Spectrum Disorder

Autism Spectrum Disorder (ASD) is a pervasive developmental disorder. DSM-5 has clubbed autistic disorder, Asperger's disorder, childhood disintegrative disorder and the general diagnosis of pervasive developmental disorder not otherwise specified under the umbrella term of ASD.

\footnotetext{
${ }^{1}$ Research Scholar, Amity Institute of Psychology and Allied Sciences, Amity University, Uttar Pradesh, Noida

${ }^{2}$ Assistant Professor, Amity Institute of Psychology and Allied Sciences,Amity University, Uttar Pradesh, Noida *Responding Author

(C) 2016 I S Gour, N Pandey; licensee IJIP. This is an Open Access Research distributed under the terms of the Creative Commons Attribution License (http://creativecommons.org/licenses/by/2.0), which permits unrestricted use, distribution, and reproduction in any Medium, provided the original work is properly cited.
} 


\section{Coping Mechanisms and Parental Relationship in the Families of a Child with Autism Spectrum Disorder}

The term spectrum implies that even though the symptoms are relatively common among the disorders, they differ on the scale of how debilitating the symptoms are and the degree to which they are manifested.

Having a child with ASD is usually associated with marital maladjustment and family dysfunction, especially in family oriented cultures (Gau et al, 2012), along with a higher rate of parental distress (White et al, 2013). While it's a given that parental stress is higher in parents with children with disability (Watt and Wagner, 2013), in patriarchal societies like India, the burden of responsibility falls mostly on the mother (Minhas et al, 2015). Families usually come up with coping mechanisms to deal with the distress of having a child diagnosed with ASD in the family and the associated social stigma as well (Divan et al, 2012). Finding coping mechanism that work well for their particular family is usually an uphill task for most parents. This requires parent to parent support (Samadi, McConkey and Kelly, 2012) and an emphasis on communication and acceptance between theparents and the professionals entrusted with the child (Danesco, 1997). Understanding the role culture plays in dictating the route of treatment for the child is important before the caretaking is streamlined between the parents and the professionals (Donald, 2015).

Coping mechanisms are needed to ensure that the parents of children with ASD can deal with day-to-day stress in a viable manner. Parenting a child with ASD is a challenging job for the parents. They deal with the added stress and responsibility in many ways and sometimes come up with specific and unique coping mechanisms that work for their family. Understanding what they're dealing with and the amount of awareness and knowledge they have about ASD also helps for forming the constantly evolving coping techniques. The three core categories for coping mechanisms are adjusting to self-change, developing treatments for the autistic child and seeking support. (Lin, Tsai and Chang, 2008). Maternal coping, for instance, has been found to be a predictor of stress of mothers with children with intellectual disabilities (John, 2012). Problem focused coping strategies usually lead to better emotional wellbeing and family function for the parents (Samadi et al, 2013).

The relationship between the parents plays a huge role in dictating the coping mechanisms the family adopts and the stress level associated with them. The quality of life of the family depended, not surprisingly, on income and the age of the child with autism (Hsiao, 2013). Psychological acceptance, as mentioned earlier, needs to be the first step before forming coping mechanisms to cater to the family. Mindful parenting is an important aspect as well and a major coping mechanism (Jones et al., 2014).

\section{METHODOLOGY}

The study was conducted in the National Capital Region (NCR) of India, spanning New Delhi, Gurgaon, Noida and Greater Noida. This study was a pilot study for families of children diagnosed with ASD and aimed to answer the following research questions- 


\section{Coping Mechanisms and Parental Relationship in the Families of a Child with Autism Spectrum Disorder}

- What are the coping mechanisms used by the families of a child with ASD?

- How were the parental relationships in these families?

- How successful were the coping mechanisms employed?

\section{Participants -}

The participants for this study were recruited at hospitals and primary health care centers and the sampling was purposive. A total sample of 10 families, with 4 members each, was taken into consideration. The inclusion criteria for selection for the families were that the families had 2 children, the younger one of whom had a diagnosis of ASD. Families with more than 2 children, or families with relatives other than parents staying with them were excluded. The families were based also on the criteria that the child diagnosed with ASD was between 5 to 12 years of age.

\section{Data Collection -}

The data for the study was generated by in-depth interviews conducted by a graduate level research scholar. All participants gave consent for the interviews. They also gave consent for transcribing the interviews. In addition, the participants were also assured of the confidentiality of the their names and details. The interview guide contained questions probing of themes of the relationship between the parents and the coping mechanisms used by them.

\section{Data Analysis -}

The interviews were face to face and conducted using the interview guide containing open-ended questions. The parents were interviewed separately. The answers were transcribed verbatim into a word processor. The source material was then assigned codes and the redundant words were removed. Additional words were added for the sole purpose of improving readability where required. Content analysis of the transcripts was then done to generate themes for the coping mechanisms manually using phenomenological psychology to interpret the meaning of the life experiences and situations of the parents. The important empirical data like the frequency of the keywords for the coping mechanisms was also analyzed for drawing conclusions of the frequency of use of a particular coping mechanism.

\section{In-depth questionnaire -}

\section{Coping mechanisms -}

Icebreaker - Tell me about your family. How many members are there? What are the names of the children?

1. When did you first get the ASD diagnosis? What did you know about ASD before that?

2. What did you think was the reason for the differences in the child?

3. What was your first reaction to the diagnosis?

4. What did you do after the initial diagnosis?

5. What were the reactions of family and friends? How do they behave with the child?

6. What were the major problems you faced socially? (Probe for stigma, social problems) 
7. What are the most pleasant and the most unpleasant experiences in taking care of the child?

8. How do you deal with the bad days?

9. What would you say is your biggest support system?

\section{Parental relationship}

1. Who noticed that there might be something different about the child first?

2. Did both of you accept the diagnosis at the time?

3. How did you two decided who take over what responsibility for the child?

4. So far, has there been a shift in expectations from each other? Why do you think that is?

5. Has there been a difference in opinion about the caretaking methods? Have there been any significant problem points between you two about this over the years?

\section{RESULTS}

The following sections describe the results of the analysis of the in-depth interviews.

\begin{tabular}{|l|l|}
\hline Coping Mechanism & No. of families using the mechanism \\
\hline Internet research & 8 \\
\hline Household help & 5 \\
\hline Professionals & 10 \\
\hline General optimism & 4 \\
\hline Family and community support & 6 \\
\hline Spirituality & 6 \\
\hline
\end{tabular}

Table 1

\section{Coping Mechanisms}

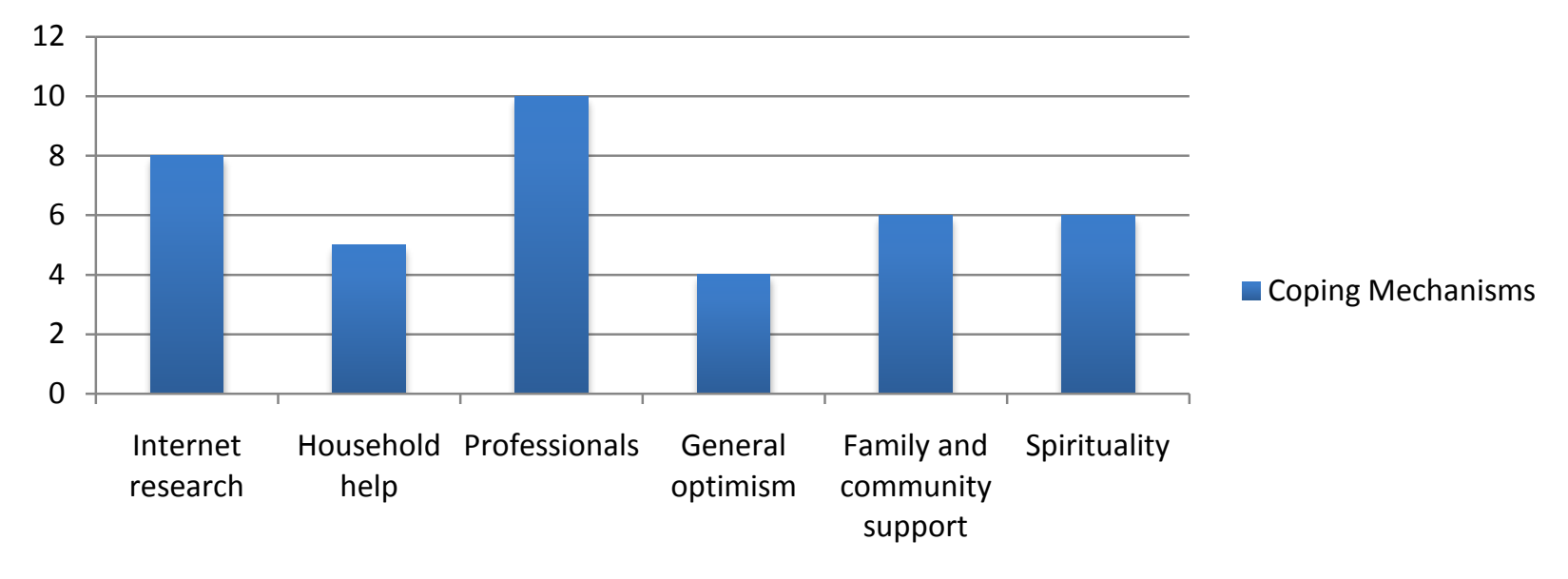

Figure 1 - Coping mechanisms employed by the families 


\section{Coping Mechanisms and Parental Relationship in the Families of a Child with Autism Spectrum Disorder}

\section{Coping Mechanisms}

The families were asked to describe the coping mechanisms they used to deal with the stress of parenting a child diagnosed with ASD and what their support systems comprised of (Figure 1, Table 1). The major coping mechanisms that the parents depended on are described below-

Internet research - The families with access to Internet relied heavily on the information available on the internet. This method, while initially extremely beneficial for the parents, eventually turned into a roadblock. Most parents were unaware about Autism Spectrum Disorder and what it meant when their child was first diagnosed. The dearth of information was fixed by researching on the internet and availing the readily available information online. However, this solution to the problem of lack of information turned into another problem altogether. Suffused with so much information online, not all of it from legitimate sources or accurate, has led to increased distress among parents and conflicts with the professionals and doctors. Owing to the freedom of posting anything online, parents often confused opinions for expertise, which was counterintuitive for the treatment. Reading about 'miracle cures' or 'child prodigies with ASD' also led to parents holding excessive and unrealistic expectations for the capabilities of the child.

Professionals - All parents interviewed depended heavily on professionals for the care and treatment of the child. While the abovementioned internet research caused clashes among parents and the professionals in regards to the care taking methods, all parents agreed unanimously that the professionals' sessions and direction helped deal with both accepting the diagnosis and dealing with the pressure of parenting a child with ASD. Going to professionals regularly also helped put things into perspective by meeting other families under similar circumstances. Going to professionals also helped underline the definition of what aberrant behavior comprised of for each child and to affix a certain appropriate reaction for that aberrant behavior. Since children diagnosed with ASD function better under a specific routine and sameness, this regulation in reaction to specific behavior turned out to be an especially helpful coping mechanism, particularly on the 'bad days' described by the parents. It helped if the treatment was tailor made for the specific child and his individual needs rather than a general system to cater to every child.

Household help - Especially in a metropolitan area like Delhi, and in cases where both parents were working, household help was considered essential for taking care of the autistic child. Despite the added expense, the parents agreed that having somebody to take care of the child helped them give more time to the typically developing sibling and deal with everyday pressures. Some mothers also reported it was easier to deal with the social stigma if they didn't actually have to go out with the child personally every time.

General optimism - In cases where the parents had a more optimistic outlook, albeit realistic at the same time, the coping was found to be much easier and better for the relationship between the members of the family. The parents reported hope for the future in terms of simple things like being able to function relatively independently, under minimal supervision, for an extended 


\section{Coping Mechanisms and Parental Relationship in the Families of a Child with Autism Spectrum Disorder}

amount of time, rather than more complex goals that other parents seemed to want like autonomy in everyday functioning. While this degree of optimism also depended on the severity of the symptoms of the child, it was generally inherent in the parents' perspective.

Family and community support - Families, extended families and relatives were another source of support for the families of the child with ASD. While initially the parents, in particular the mother, exhibited trepidation to entrust their child with special needs to somebody else, in most cases, the support was later considered to be a major boost for the coping with the child. For parents who lived in smaller communities, the community support was paramount. The typically developing siblings, especially in the case of elder sisters, also took a proactive role in taking over the responsibility of taking care of the child and in some cases, took special courses to learn how to properly deal with children with ASD.

Spirituality - Spirituality was also mentioned as a major coping mechanism for the culture oriented families of India. The definition of spirituality in these cases, spanned a broad spectrum from holding special prayer ceremonies regularly to simply visiting temples to atone for 'past life sins'. This mechanism served as a comforting ritual as well as a coping mechanism for the families.

\section{Relationship between parents}

A lot of factors contributed to the nature of relationship between parents. Interviewing the parents separately, rather than as a couple highlighted the gap in communication between most parents. The main reason for the stress in the relationship seemed to be rooted in difference in opinion in the caretaking methods. There was a paradox concerning the household help for the mothers, wherein the added expenses of having a child with ASD required both parents to work, but the working mothers reported even more guilt for leaving the child with household help. This issue of who took over the responsibility for the child was where the difference in opinion lay. While mothers reported a need for the household help, even with the aforementioned added guilt, for diffusion of responsibility, the fathers held an opinion that the mothers needed to be there personally for the child instead of delegating the responsibility.

Most parents reported a shift in responsibility from the time the initial diagnosis was made to when this interview occurred. When first diagnosed, almost all parents reacted with denial. Even though the symptoms were clear enough to make a diagnosis, it was after a particular amount of time that the parents accepted the diagnosis. A few years after the diagnosis, the demarcation of roles became clear for the parents. While the parents usually mutually agreed upon the roles at the time, this also was a cause of rift for the parents. The mothers described it as a never-ending cycle of events that felt exhausting and which became another reason for the miscommunication between the mother and father. The parental relationship also suffered at the helm of the typically developing siblings. One parent usually put too much pressure on the sibling, defined as overcompensation by the other parent and it caused distress among the entire family. This makes 


\section{Coping Mechanisms and Parental Relationship in the Families of a Child with Autism Spectrum Disorder}

a vicious circle in the sense that since the routine is disrupted by the distress, the child with ASD stops functioning well in the lack of a system, which leads to further distress for the family.

\section{DISCUSSION}

The pilot study shows that the first step in coping with ASD is the acceptance of the diagnosis, which is in keeping with already existing research (Desai, Wertz and Patel, 2012). Culture was found to play a major role in the shaping of these coping mechanisms. According to a 2008 study by Lin, Tsai and Chang, the three core categories for coping mechanisms are adjusting to selfchange, developing treatments for the autistic child and seeking support. This was supported by the data in this research which delineated customized professional help and general optimism on the parents' part as one of the main coping mechanisms. Spirituality was also one of the coping mechanism, unsurprising in a country as rich in culture and tradition as India. Some parents attributed the cause of the ASD to karma as well, and it was found that spiritual practices like reading the scriptures of visiting the temple also formed basis of coping mechanisms. This also corroborates previous research which showed that positive religious coping was associated with better religious outcome and greater stress related growth (Tarakeshwar and Pargament, 2001).

As expected, with previous studies showing that parental distress and ineffective coping mechanisms have a negative effect on family functioning and the relationship between parents (Gau, 2012), this study also found that parental relationship suffers and there is a family dysfunction in parents of a child with ASD. This distress and dysfunction was found to be rooted in a lack of communication between the parents. The parents usually look to each for support (Searing, Jean and Grainger, 2013), but parental perspective of their locus of control was usually found to be a pretty strong predictor of parental stress, which also affected their coping mechanism (Jones and Passey, 2005). In this study, however, most of the parents who could afford it chose to depend on household help and delegated responsibility of the child to them. This was also a cause of concern for the fathers who in most cases described their role as the breadwinner and the mother as the primary caregiver to the child. This was also a source of disagreement between parents. In case, however, where the parents had an optimistic outlook towards the future of the autistic child also reported a more complementary relation between them and a lesser degree of family dysfunction.

\section{LIMITATIONS AND CONCLUSIONS}

The major limitation of this study is that the sampling was done in hospitals where the parents had access to health care providers and professionals. Another limitation is that even though the interviews were in depth; they still relied on the answers of the parents and not on a direct objective observation of day-to-day lives of the families. From the interviews, it can be concluded that family therapy and opening communication channels between the parents seems to be the best way to resolve this family dysfunction and coming up with a more streamlined approach towards coping with a child with ASD. The most effective coping mechanisms for the 


\section{Coping Mechanisms and Parental Relationship in the Families of a Child with Autism Spectrum Disorder}

families seem to be professional help, a general optimistic perspective, spirituality and access to accurate information.

\section{REFERENCES}

Azeem, M. W., Dogar, I. A., Shah, S., Cheema, M. A., Asmat, A., Akbar, M., ... \&Haider, I. I. (2013). Anxiety and Depression among Parents of Children with Intellectual Disability in Pakistan. Journal of the Canadian Academy of Child and Adolescent Psychiatry, 22(4), 290.

Chan, G. W., \&Goh, E. C. (2014). 'My Parents told us that they will always Treat my Brother Differently Because he is Autistic'-Are Siblings of Autistic Children the Forgotten Ones?. Journal of Social Work Practice, 28(2), 155-171.

Constantino, J. N., \&Charman, T. (2015). Diagnosis of autism spectrum disorder: reconciling the syndrome, its diverse origins, and variation in expression. The Lancet Neurology.

Cox, C. R., Eaton, S., Ekas, N. V., \& Van Enkevort, E. A. (2015). Death concerns and psychological well-being in mothers of children with autism spectrum disorder. Research in developmental

Dabrowska, A., \&Pisula, E. (2010). Parenting stress and coping styles in mothers and fathers of pre-school children with autism and Down syndrome. Journal of Intellectual Disability Research, 54(3), 266-280.

Danseco, E. R. (1997). Parental beliefs on childhood disability: Insights on culture, child development and intervention. International Journal of Disability, Development and Education, 44(1), 41-52.

DePape, A. M., \& Lindsay, S. (2015). Parents' experiences of caring for a child with autism spectrum disorder. Qualitative health research, 25(4), 569-583.

Derguy, C., Michel, G., M'bailara, K., Roux, S., \& Bouvard, M. (2015). Assessing needs in parents of children with autism spectrum disorder: A crucial preliminary step to target relevant issues for support programs. Journal of Intellectual and Developmental Disability, 40(2), 156-166.

Desai, M. U., Divan, G., Wertz, F. J., \& Patel, V. (2012). The discovery of autism: Indian parents' experiences of caring for their child with an autism spectrum disorder. Transcultural psychiatry, 49(3-4), 613-637.

Divan, G., Vajaratkar, V., Desai, M. U., Strik-Lievers, L., \& Patel, V. (2012).Challenges, coping strategies, and unmet needs of families with a child with autism spectrum disorder in Goa, India. Autism Research, 5(3), 190-200.

Dolev, S., Sher-Censor, E., Baransi, N., Amara, K., \& Said, M. (2016). Resolution of the child's ASD diagnosis among Arab-Israeli mothers: Associations with maternal sensitivity and wellbeing. Research in Autism Spectrum Disorders, 21, 73-83.

Donald, K. (2015). Understanding Parent Perceptions of Autism Spectrum Disorder Diagnosis and Intervention Services Through A Culturally Responsive Framework (Doctoral dissertation, Concordia University). 


\section{Coping Mechanisms and Parental Relationship in the Families of a Child with Autism Spectrum Disorder}

Estes, A., Munson, J., Dawson, G., Koehler, E., Zhou, X. H., \& Abbott, R. (2009). Parenting stress and psychological functioning among mothers of preschool children with autism and developmental delay. Autism, 13(4), 375-387.

Frantzen, K. K., Lauritsen, M. B., Jørgensen, M., Tanggaard, L., Fetters, M. D., Aikens, J. E., \&Bjerrum, M. (2015). Parental Self-perception in the Autism Spectrum Disorder Literature: a Systematic Mixed Studies Review. Review Journal of Autism and Developmental Disorders, 1-19.

Gau, S. S. F., Chou, M. C., Chiang, H. L., Lee, J. C., Wong, C. C., Chou, W. J., \& Wu, Y. Y. (2012). Parental adjustment, marital relationship, and family function in families of children with autism. Research in Autism Spectrum Disorders, 6(1), 263-270.

Heer, K. (2012). The cultural context of care-giving: a phenomenological exploration of the experiences of South Asian carers caring for children with intellectual/developmental disabilities (Doctoral dissertation, University of Birmingham).

Heiman, T. (2002). Parents of children with disabilities: Resilience, coping, and future expectations. Journal of developmental and physical disabilities,14(2), 159-171.

Higgins, D. J., Bailey, S. R., \& Pearce, J. C. (2005). Factors associated with functioning style and coping strategies of families with a child with an autism spectrum disorder. Autism, 9(2), 125-137.

Hsiao, Y. J. (2013). Parental Stress, Family-Professional Partnerships, and Family Quality of Life: Families of Children with Autism Spectrum Disorder. disabilities, 45, 229-238.

Hutton, A. M., \& Caron, S. L. (2005). Experiences of families with children with autism in rural New England. Focus on autism and other developmental disabilities, 20(3), 180-189.

Jegatheesan, B., Fowler, S., \& Miller, P. J. (2010). From symptom recognition to services: how South Asian Muslim immigrant families navigate autism. Disability \& Society, 25(7), 797-811.

John, A. (2012). Stress among mothers of children with intellectual disabilities in urban India: role of gender and maternal coping. Journal of Applied Research in Intellectual Disabilities, 25(4), 372-382.

Jones, J., Passey, J. (2005). Family adaptation, coping and resources: Parents of children with developmental disabilities and behaviourproblems. Journal on Developmental Disabilities, 11(1), 31-46.

Jones, L., Hastings, R. P., Totsika, V., Keane, L., \&Rhule, N. (2014). Child behavior problems and parental well-being in families of children with autism: the mediating role of mindfulness and acceptance. American journal on intellectual and developmental disabilities, 119(2), 171-185.

Lin, C. R., Tsai, Y. F., \& Chang, H. L. (2008). Coping mechanisms of parents of children recently diagnosed with autism in Taiwan: a qualitative study. Journal of clinical nursing, 17(20), 2733-2740.

Luther, E. H., Canham, D. L., \&Cureton, V. Y. (2005). Coping and social support for parents of children with autism. The Journal of School Nursing,21(1), 40-47. 


\section{Coping Mechanisms and Parental Relationship in the Families of a Child with Autism Spectrum Disorder}

Malhotra, S., Khan, W., \& Bhatia, M. S. (2012).Quality of life of parents having children with developmental disabilities. Delhi Psychiatry Journal,15(1), 173-174.

Mancil, G. R., Boyd, B. A., \&Bedesem, P. (2009). Parental stress and autism: are there useful coping strategies?. Education and Training in Developmental Disabilities, 523-537.

McConkey, R., \&Samadi, S. A. (2013). The impact of mutual support on Iranian parents of children with an autism spectrum disorder: a longitudinal study. Disability and rehabilitation, 35(9), 775-784.

Minhas, A., Vajaratkar, V., Divan, G., Hamdani, S. U., Leadbitter, K., Taylor, C., ... \& Green, J. (2015).Parents' perspectives on care of children with autistic spectrum disorder in South Asia-Views from Pakistan and India.International Review of Psychiatry, 27(3), 247-256.

Myers, B. J., Mackintosh, V. H., \&Goin-Kochel, R. P. (2009). "My greatest joy and my greatest heart ache:" Parents' own words on how having a child in the autism spectrum has affected their lives and their families' lives.Research in Autism Spectrum Disorders, 3(3), 670-684.

Nolcheva, M., \&Trajkovski, V. (2015). Exploratory study: stress, coping and support among parents of children with autism spectrum disorders. Journal of Special Education and Rehabilitation, 16(3-4), 84-100.

Pathappillil, J. S. J. (2011). Through Our Eyes: A Qualitative Study of Indian Mothers and Their Perceptions of Autism.

Samadi, S. A., McConkey, R., \& Kelly, G. (2012). The information and support needs of Iranian parents of children with autism spectrum disorders. Early Child Development and Care, 182(11), 1439-1453.

Samadi, S. A., McConkey, R., \& Kelly, G. (2013). Enhancing parental well-being and coping through a family-centred short course for Iranian parents of children with an autism spectrum disorder. Autism, 17(1), 27-43.

Searing, B. M. J., Graham, F., \& Grainger, R. (2015). Support Needs of Families Living with Children with Autism Spectrum Disorder. Journal of autism and developmental disorders, 45(11), 3693-3702.

Siklos, S., \& Kerns, K. A. (2006). Assessing need for social support in parents of children with autism and Down syndrome. Journal of autism and developmental disorders, 36(7), 921933.

Sikora, D., Moran, E., Orlich, F., Hall, T. A., Kovacs, E. A., Delahaye, J., ... \&Kuhlthau, K. (2013).The relationship between family functioning and behavior problems in children with autism spectrum disorders. Research in Autism Spectrum Disorders, 7(2), 307-315.

Sivberg, B. (2002). Family System and Coping Behaviors A comparison between parents of children with autistic spectrum disorders and parents with non-autistic children. Autism, 6(4), 397-409.

Tarakeshwar, N., \&Pargament, K. I. (2001). Religious coping in families of children with autism. Focus on Autism and Other Developmental Disabilities,16(4), 247-260. 


\section{Coping Mechanisms and Parental Relationship in the Families of a Child with Autism Spectrum Disorder}

Twoy, R., Connolly, P. M., \& Novak, J. M. (2007). Coping strategies used by parents of children with autism. Journal of the American Academy of Nurse Practitioners, 19(5), 251-260.

Uljarević, M., Prior, M. R., \& Leekam, S. R. (2014). First evidence of sensory a typicality in mothers of children with Autism Spectrum Disorder (ASD).Molecular autism, 5(1), 26.

Watt, M., \& Wagner, S. L. (2013). Parenting a child with autism spectrum disorder: parental work context. Community, Work \& Family, 16(1), 20-38.

White, T. J., Perry, D. F., Biel, M. G., \& Jacobstein, D. M. (2013).Assessment and Documentation of Parental Distress as Evidenced in Clinical Reports Prepared by the Georgetown University Hospital Autism and Communication Disorders Clinic (ACDC). Issues, 7(1). 